\title{
Rechnungswesenunterricht am Scheideweg? - Einführung in den Sammelband
}

\author{
Jürgen Seifried und Detlef Sembill
}

\section{Zur Kritik am herkömmlichen Rechnungswesenunterricht}

Vor dem Hintergrund des ökonomischen und technologischen Wandels und der daraus resultierenden verănderten Anforderungen an komplexe Lehr-Lern-Arrangements gerät im kaufmännischen Schulwesen insbesondere der tradierte Rechnungswesenunterricht vermehrt in die Kritik, Schwachstellen und Grenzen des herkömmlichen Rechnungswesenunterrichts werden aufgezeigt (siehe zusammenfassend Preiß 2000 und Seifried 2004a sowie die dort jeweils zitierte Literatur). Die fachdidaktische Auseinandersetzung zwischen Lehrkräften, Vertretern der Studienseminare und Schulbuchautoren belegt die um sich greifende Unzufriedenheit. Die vielfältigen Beanstandungen an der traditionellen Vorgehensweise können vornehmlich zwei Punkten zugeordnet werden:

- Das dem System der Buchführung zugrunde gelegte Erklärungsmodell Viele Kritiker des herkömmlichen Rechnungswesenunterrichts weisen darauf hin, dass die Ausrichtung an der Bilanzmethode - das bis heute in der Unterrichtspraxis vorherrschende didaktische Modell - als problematisch zu kennzeichnen ist (siehe z. B. die Beitrăge in Preiß \& Tramm 1996 sowie Preiß 1999). Insbesondere wird bemängelt, dass in der Schulbuchführung die Bedeutung der Unterscheidung von Buch- und Ist-Werten nicht in ausreichendem Maße thematisiert wird. Bei einer impliziten Gleichsetzung von Ist- und Soll-Daten (in vielen Lehrbüchern finden sich Hinweise wie „Buchwerte und Inventurwerte stimmen überein") wird unterstellt, dass de Gewinn allein anhand der Daten der Buchführung ermittelt werden kann. Die Frage nach den Ursachen von Inventurdifferenzen sowie deren Ausgleich mittels Korrek turbuchungen fällt somit unter den Tisch; der Fehlinterpretation der Bilanz als eine Ansammlung von Kontensalden wird Vorschub geleistet. Diese Vorgehensweise verstellt den Blick auf die bei der Bilanzerstellung offen stehenden Entscheidungsspielräume (z. B. hinsichtlich der Bewertung von Vermögensgegenständen). Nicht zuletz aus diesen Gründen findet sich die explizite Forderung nach der Abstimmung zwi- 
schen Inventurdaten und den Ergebnissen der laufenden Buchführung mittlerweile in einschlägigen (Rahmen-)Lehrplänen wieder. - Komplexität und Authentizität von Lehr-Lern-Situationen sowie Ausmaß und Art
der Lehrerunterstützung

Aus unterrichtsmethodischer Sicht konzentriert sich die Kritik vornehmlich auf die lehrerzentrierte Ausgestaltung der Lehr-Lern-Prozesse. Die Beanstandungen richten sich dabei gegen eine in kleine Lernschritte gegliederte, stark lehrergesteuerte Vorgehensweise. Hohe Vergessensraten, intellektuelle Unterforderung der Lernenden, fehlendes Verständnis für die Gesamtzusammenhänge sowie Defizite hinsichtlich wünschenswerter Qualifikationen wie Problemlösefähigkeit und Handlungskompetenz werden in diesem Zusammenhang angeführt. Erschwerend kommt hinzu, dass die einzelnen Lehreinheiten weitgehend identisch aufgebaut und Methoden nur selten variiert werden.

Die oben vorgenommene Trennung zwischen Kritikpunkten fachdidaktisch-curricularer und fachdidaktisch-methodischer Natur darf nicht darüber hinwegtäuschen, dass die skizzierten Problemlagen eng zusammenhängen. Dies belegt auch der Blick auf mögliche Ursachen von Lernschwierigkeiten im Rechnungswesenunterricht. Onne eine verstärkte Problemorientierung kommt der Ausweitung der Schüleraktivität Alibicharakter zu; und wenn Schüler über mehrere Unterrichtsstunden hinweg eigenständig lernen sollen, müssen statt Aufgaben ohne Problemgehalt echte, nicht wohl-definierte Probleme dargeboten und die Eigenaktivităt der Lernenden angeregt und unterstützend begleitet werden. Bei aller wünschenswerten Hinwendung zur Problemorientierung sei jedoch davor gewarnt, die Einübung der Techniken der Buchführung zu vernachlässigen. Eine ungenügende Beherrschung der Methoden des Rechnungswesens führt dazu, dass Lernende nicht in der Lage sind, Datenanalysen und Interpretationen ohne Schwierigkeiten durchzuführen (Dubs 1996, S. 132). Die Beherrschung der für die Bearbeitung komplexer Problemstellungen erforderlichen Grundfertigkeiten stellt letztlich eine notwendige - wenn auch nicht hinreichende Bedingung für die Fähigkeit zur Datenanalyse und Dateninterpretation dar. Bei der Konkretisierung der curricularen Rahmenbedingungen im Zuge der Konstruktion vernetzter Problemstellungen kommt daher auch der Einbindung variantenreicher Übungs- und Vertiefungsphasen hohes Gewicht zu.

Zusammenfassend lässt sich feststellen, dass sich die curriculare Ausrichtung der "Schulbuchführung" mittlerweile sowohl von den Anforderungen der Praxis als auch von den jeweiligen Bezugswissenschaften so weit entfernt hat, dass eine inhaltliche Neuausrichtung unumgănglich erscheint. Trotz vielfăltiger Kritik wird die Auswahl und Anordnung der Lerninhalte von Unterrichtspraktikern jedoch nur selten in Frage gestellt: Bei einer Befragung von elf Fachleitern niedersăchsischer Studienseminare konnten Tramm, Hinrichs \& Langenheim (1996, S. 198 ff.) Anhaltspunkte dafür finden, dass nicht in der Stoffauswahl, sondern in erster Linie in der methodischen Gestaltung des Unterrichts Potenzial zur Steigerung der Unterrichtsqualităt gesehen wird. Diese Sicht der Dinge erscheint recht einseitig, denn bei genauer Betrachtung zwingt das herkömmliche Vorgehen den Lehrenden zu Konstruktionen, die das betriebswirtschaftliche Verständnis erheblich erschweren. Auch sind nur wenige Reformbemühungen auszumachen, vielmehr kann man den Eindruck gewinnen, als habe die Diskussion um handlungsorientierten Unterricht einen weiten Bogen um den Buchführungsunterricht gemacht).

Ungeachtet der mannigfaltigen Monita an der überlieferten Qualifizierungspraxis scheint die Reformbereitschaft in der schulischen Praxis nicht hinreichend ausgeprägt zu sein. Bei einer Durchsicht der Beiträge zur Didaktik des Rechnungswesens ziehen Reinisch \& Struve (2002, S. 108) folgendes - nur wenig ermutigendes - Fazit: Während Buchführung bei Lernenden als schwierige (und wohl auch als "trockene") Materie gelte, sei das Fach bei Lehrkräften "angesichts der ausgearbeiteten Fachdidaktik, der klar strukturierten Lehrpläne und der langjährig eingeführten Schulbücher" sehr beliebt und werde als "verteidigungswürdige Domăne" betrachtet. Bei Fachdidaktikern, die eine didaktisch-curriculare Innovation des Buchführungsunterrichts anstreben, gelte das Fach als "schwer zu knackende Nuss". Sie klagen über die "Reformresistenz" dieses Faches und das "Beharrungsvermögen traditionellen didaktischen Denkens in der Unterrichtspraxis." Kurz gefasst: Forschergruppen, die eine Umgestaltung der überlieferten Lehr-Lern-Praxis zum Ziel haben, stoßen auf vergleichsweise schwierige Rahmenbedingungen.

Passend zu obigen Aussagen kann konstatiert werden, dass eine Diskussion über das Verhältnis von Lerninhalt und methodischer Konzeption der Lehr-Lern-Prozesse für den Rechnungswesenunterricht bisher weitgehend unterblieb. Es gilt offenbar folgende Maxime: Der Inhalt bestimmt die Methode. Die pädagogischen Bemühungen konzentrieren sich in erster Linie auf die Frage, wie vorgegebene Inhalte, die eine bestimmte innere Ordnung und Struktur aufweisen, den Lernenden vermittelt werden können. Die Aufgabe der Lehrperson besteht dann im Transport der Lerninhalte; die Sachstruktur soll so vermittelt werden, dass sie von den Schülern korrekt, voliständig 
und überdauernd erfasst wird. Die dieser Position zugrunde liegenden Prämissen sind mit Terhart (1997, S. 43) aufs Schärfste zu kritisieren: Weder die "naiv-realistische, ontologische Auffassung" von einer gegebenen inneren Ordnung von Lerninhalten noch das Bild eines passiven Aufnehmens von Inhalten ist haltbar. Versteht man den Lerner als aktiv-aneignendes, entwicklungsfähiges Subjekt (Sembill 1992), so muss statt des bisher vorherrschenden "Inhaltsdogmatismus bei gleichzeitiger Verkürzung der Methodenproblematik auf Verfahrensfragen" "(Terhart 1997, S. 44) eine sachlich-konstruktive Auseinandersetzung mit den bestehenden Interdependenzen zwischen Lerninhalten und methodisch-medialen Gesichtspunkten (Tramm 1992) in Gang gebracht werden. Im vorliegenden Sammelband finden sich diesbezüglich vielfältige Hinweise.

\section{2 Ökonomische Kompetenz durch Rechnungswesenunterricht}

Auch wenn man sich für den Rechnungswesenunterricht bisweilen mehr Praxisnähe wünscht, reicht es sicherlich nicht aus, Qualifizierungsbemühungen an den Anforderungen betrieblicher Funktionsbereiche auszurichten. Ebenso führt die Fokussierung auf die Interpretation ökonomischer Sachverhalte nicht zu dem gewünschten Kompetenzprofil, wenn man die Vermittlung von Rechen- und Buchungstechniken vernachlässigt und die Lernenden keine Kenntnis darüber besitzen, wie die zu interpretierenden Daten zustande kommen. Als Leitziel des Rechnungswesens auf der curricularen Ebene ist daher das "Verstehen ökonomischer Zuşammenhänge über die Durchdringung und Beherrschung kaufmännischer Techniken" (Tramm \& Preiß 1996, S. 5), also die "Verbindung von Handlungssicherheit und Verständnis" (Schneider 2000, S. 200) zu verfolgen. Im Lernbereich "Rechnungswesen" sollte es, so Reetz \& Tramm (2000, S. 108), primär darum gehen, "den Lernenden ein Instrumentarium und eine kognitive Perspektive zu vermitteln, die es ihnen ermöglichen, ökonomische Systemzusammenhänge und Handlungsweisen aus einer originär wirtschaftlichen Perspektive zu verstehen und auf dieser Basis auch für die Planung, Realisation und Bewertung eigenen kaufmännischen Handelns zu nutzen". Schlicht gesagt lässt sich das als Forderung nach der Ausbildung von Handlungskompetenz interpretieren. Dabei ist der Kompetenzbegriff umfassend zu verstehen, er schließt emotionale, motivationale und ethische Aspekte ein und zielt auf übergreifende Fähigkeiten wie Problemlösen, Kommunikations- und Teamfähigkeit ab (Achtenhagen 1996, siehe auch Sembill 1992).
Aus den obigen Ausführungen lässt sich ableiten, dass es bei einer Umgestaltung des Rechnungswesenunterrichts nicht genügt, lediglich auf eine kognitive Aufwertung des herkömmlichen Unterrichts abzustellen und eine Anreicherung des traditionellen Inhaltskanons ( $z$. B. um dispositive Elemente) zu verfolgen. Bei den Überlegungen zur didaktischen Konzeption des Rechnungswesenunterrichts sollte man vielmehr von Anfang an auch emotional-motivationale Kriterien ins Kalkül ziehen und auf ein selbsttätiges und sinnverstehendes Lernen in komplexen Situationszusammenhängen abzielen. Diese integrierende Betrachtungsweise stellt darauf $a b$, dass nur bei einer simultanen Berücksichtigung unterrichtsmethodischer und curricularer Gestaltungsoptionen eine nachhaltige Steigerung der Unterrichtsqualität möglich wird. Aus unterrichtsmethodischer Sicht könnte insbesondere selbstorganisationsoffener Unterricht als Konzeption mit Lehr-Lern-Arrangements, die einen herausragenden Anteil selbstorganisierten Lernens zulassen, einen geeigneten Rahmen bieten. Bei diesem Lehr-Lern-Arrangement wird explizit berücksichtigt, dass neben Erwartungen auch emotionale Prozesse mit dem Handeln und Problemlösen verknüpft sind. Im Zentrum des Selbstorganisierten Lernens steht das so genannte Grundprinzip geplanten Handelns, das die für einen kompletten Problemlöseprozess notwendigen Handlungsschritte beschreibt und auf der Grundlage der Prämissen einer integrativen Menschenbildannahme und subjektiven Handlungsrelevanz die Disponibilităt von Handlungszielen sowie die Bedeutung interner, aktiver Bewertungsprozesse ausdrücklich herausstellt (Sembill 1992, S. 108 ff.). Wie empirische Untersuchungen belegen, stellt die Gestaltung einer selbstorganisationsoffenen Lernumgebung (Sembill 1992, 2000, 2004) auch für das Rechnungswesen eine Erfolg versprechende Möglichkeit dar, um eine schüleraktivere Unterrichtsgestaltung zu realisieren. Angesichts der Passung grundlegender Gestaltungsprinzipien scheint insbesondere in der Kombination des Selbstorganisierten Lernens mit dem wirtschaftsinstrumentellen Rechnungswesen ein möglicher Ansatz zur Steigerung der Unterrichtsqualităt zu liegen (Seifried, Brouër \& Sembill 2002; Seifried 2004a, 2004b; Seifried \& Sembill 2004).

\section{Zum Stand der fachdidaktischen Diskussion}

Nachdem der Rechnungswesenunterricht von den universitären Vertretern der Wirtschaftspădagogik lange Zeit nahezu stiefmütterlich behandelt wurde (Reinisch \& Struve 2002, S. 107), hat sich das Bild in den letzten fünfzehn Jahren gewandelt: Hinsichtlich der Auseinandersetzung mit der Didaktik des Rechnungswesens aus historischer Sicht ist insbesondere die Habilitationsschrift von Reinisch (1991) her- 
vorzuheben. Mit den von Achtenhagen (1990) und Preiß \& Tramm (1996) herausgegebenen Sammelbänden, der Monographie von Sloane (1996) sowie der Dissertation von Preiß (1999) hat die Wirtschaftspädagogik die Diskussion um den Rechnungswesenunterricht aufgegriffen. "Marktführer" in Österreich ist der didaktische Ansatz von Schneider (2000), dessen Lehrbuch „Betriebliches Rechnungswesen für Einsteiger" mittlerweile in der vierten Auflage (2002) erhältlich ist. Bei den genannten didaktische didaktische Konzepte oder Modelle für den Rechnungswesenunterricht dargestellt und begründet

Insbesondere mit der Didaktik des wirtschaftsinstrumentellen Rechnungswesens (Preiß \& Tramm 1990, 1996; Preiß 1999, 2000) liegt bezüglich der Auswahl und Sequenzierung von Lerninhalten ein vielversprechender und mittlerweile in die Unterrichtspraxis umgesetzter Ansatz vor. Trotz einer prinzipiell als handlungsorientiert zu kennzeichnenden Grundausrichtung konzentriert sich dieses Konzept in erster Linie auf curriculare Problemfelder. Anknüpfend an den Göttinger Entwicklungsarbeiten beschäftigt sich die Bamberger Wirtschaftspädagogen u. a. mit der Frage, wie der lehrerzentrierten Ausrichtung der Lehr-Lern-Prozesse entgegenzuwirken ist und die Lernenden angemessen an der Planung, Durchführung und Kontrolle ihrer Lernaktivităten beteiligt werden können. In diesem Zusammenhang wurde mittels aufwendiger empirischer Untersuchungen gezeigt, dass insbesondere in der Kombination des wirtschaftsinstrumentellen Rechnungswesens mit der Konzeption des selbstorganisierten Lernens nach Sembill ein vielversprechender Ansatz zur Steigerung der Unterrichtsqualität liegen könnte (s. o.).

Die Beiträge des vorliegenden Sammelbandes greifen die skizzierten Diskussionslinien auf und vertiefen diese. Im Beitrag von Holger Reinisçh geht es aus historischer Sicht um zentrale Leitlinien in der Diskussion des Rechnungswesenunterrichts. Er unterscheidet im Rahmen der Kontroverse um das "richtige" Leitbild für den Rechnungswesenunterricht zwischen einem "denkenden" und einem „praktischen" Buchhalter. Eine am Konzept des „praktischen" Buchhalters orientierte Ausbildung vertritt die Auffassung, dass die im Unterricht angeeigneten Fertigkeiten und Fähigkeiten in der betrieblichen Praxis nutzbar sein müssen. Diese prinzipielle Verengung des Anforderungsprofils auf ein Bündel funktionaler Qualifikationsanforderungen, die Ankünftige Kaufleute beherrschen sollen geht mit kanons einher: Es Kenntnisse und Fertigkeiten vermittelt Kenntnisse und Fertigkeiten vermittelt werden, die den jeweiligen Praxisanforderun- gen genügen. Demgegenüber kommt einer systematischen geistigen Durchdringung sowie dem Verständnis der Systematik der doppelten Buchführung lediglich ein untergeordneter Stellenwert zu. Prinzipiell wird hier von einer ausführenden und nicht von einer dispositiven Tätigkeit ausgegangen. ${ }^{1}$

Auch beim Leitbild des „denkenden" Buchhalters bildet die betriebliche Praxis einen wichtigen Bezugspunkt. Im Unterschied zum „praktischen Buchhalter" wird bei dieser Argumentationslinie das berufliche Handeln von Kaufleuten jedoch als permanenter, auf quantitativ aufbereiteten Daten basierender Entscheidungsprozess in einer komplexen und dynamischen Umwelt verstanden. Dieses modifizierte Praxisverständnis führt zu einer Ausweitung der an kaufmännische Angestellte gerichteten Qualifikationsanforderungen um dispositive Elemente; die pädagogischen Anstrengungen zielen ab auf das kompetente und verantwortliche ökonomische Handeln sowohl im betrieblichen als auch im privaten Kontext. Gleichzeitig strebt man die Verknüpfung des Rechnungswesenunterrichts mit anderen betriebswirtschaftlichen Teilbereichen an (Reinisch 1996, S. 54 f.; Tramm \& Preiß 1996, S. 1 ff.). Diese Einsichten sind nach wie vor aktuell, aber keinesfalls neu. Reinisch zeigt insbesondere unter Rückgriff auf die noch heute bemerkenswerten Überlegungen von Philipson (1813) eindrucksvoll, welche Lehren aus historischen Schriften für die Aufgabe der didaktischen Konstruktion von lernergerechten Lernsituationen gezogen werden können (bzw. könnten)

Die Auseinandersetzung um die "richtige" Methode im Buchführungsunterricht, der in den Beiträgen von Schneider, Preiß, Tramm sowie Seifried, Wolf, Klüber \& Sembill eine zentrale Rolle zukommt, konzentrierte sich dann insbesondere auf drei Aspekte: Zum einen wird um das dem System der Buchführung zugrunde gelegte Erklärungsmodell gerungen. Zum anderen bestehen unterschiedliche Meinungen über das notwendige Ausmaß der Komplexität und Authentizităt von Lehr-Lern-

\footnotetext{
Der Buchhaltungsunterricht galt lange Zeit zudem als geeignetes instrument, um neben Buchhaltungstechniken auch religiös-ethische bzW. ab dem 18. Jahrhundert utilitaristische Elemente zu thematisieren Die Vorstellung, mittels Buchfuhrungsunterricht auch weltanschauliche Gesinnungs bildung betreiben zu wollen, wirkt bis weit in das 20 . Jahrhundert hinein (Reinisch 1996, S. 59 f.) Noch in den 60er Jahren des letzten Jahrhunderts wird auf von nationalsozialistischen Argumentationsketten weitgehend bereinigte Bildungswertanalysen von Buze (1936) zurückgegriffen. Dieser bestimmt den Bildungswert des Buchführungsunterrichts sowohl in der technischen Schulung ais auch in der Denk- und Willensschulung und betont dabei die Gesinnungsbildung. Die Buchhaltung sei, so Butze, "ein vorzügliches Mittel", um zu "Genauigkeit, Sauberkeit und Ordnung" zu erziehen (ebd., S. 70). "Die größte Steigerung in der Heranbildung eines persönlichen Gepräges des Menschen ist erreicht, wenn möglichst viele wertvolle Charaktereigenschaften in einem Maße vorhanden sind, daß der bewußte Wille überflussig geworden ist und der so erzogene Mensch gar nicht mehr anders kann, als fleißig, aufmerksam, sauber, gewissenhaft, ordentlich zu sein." (ebd.).
} 
Situationen und eng damit verbunden über das Ausmaß und die Art der Lehrerunterstützung.

In seinem Beitrag plädiert Wilfried Schneider für einen auf klaren Strukturen und Regeln aufgebauten Unterricht und wendet sich gegen eine Überbetonung des selbstentdeckenden und selbstgesteuerten Lernens, da hier die Gefahr von Lerndefiziten durch unzureichende Abstraktion bestehe. Als Merkmale guten Unterrichts nennt Schneider Problemorientierung und starke, langsam abnehmende Lehrerunterstützung ("fading"). Darüber hinaus liefert der Autor einen in Schulpraxis erprobten Ansatz zur Vermittlung der Grundzüge des Systems der doppelten Buchführung. Schneider schlägt vor, die Finanzrechnung eines Kleinbetriebs als Einstiegssituation zu verwenden und zeigt anhand eines Goldschmieds ( ${ }_{n}$ Goldi ${ }^{4}$ ) eine mögliche didaktische Modellierung des Anfangsunterrichts. Zentral sind hierbei die Unterscheidung von Zahlungsmittelbestand und Zahlungsfähigkeit von der Verrechnung von Vermögen und Kapital bzw. Aufwand und Ertrag.

Mit der Diskussion der Vorzüge und Nachteile der von Frank Achtenhagen und ehemaligen (Tade Tramm, Universität Hamburg) bzw. noch heute in Göttingen wirkenden Mitarbeitern (Peter Preiß) entwickelten Konzeption des wirtschaftsinstrumentellen Rechnungswesens schlägt Schneider den Bogen zu den Folgebeitrăgen von Preiß und Tramm. Der Göttinger Lehrstuhlinhaber und seine damaligen Mitstreiter legten bereits Anfang der 1990er Jahre mit der so genannten "Modellierungsmethode" im Rahmen der Didaktik des wirtschaftsinstrumentellen Rechnungswesens eine alternative Einstiegskonzeption vor, in der die Bilanz als formales Referenzmodell durch ein inhaltliches Referenzmodell ersetzt wird (siehe hierzu zusammenfassend die Beiträge in Achtenhagen 1990, Preiß \& Tramm 1996 sowie Preiß 1999). In der Schulpraxis stößt die Didaktik des wirtschaftsinstrumentellen Rechnungswesens auf große Akzeptanz. Hierzu trägt sicherlich auch bei, dass durch die Veröffentlichung eines belegorientierten Grundkurses Rechnungswesens durch Getsch \& Preiß (2001) sowie durch das Erscheinen des Lehrbuches „Wirtschaftsinstrumentelles Rechnungswesen" von Joost, Kripke \& Tramm (2002) umfangreiche Unterrichtsmaterialien zur Verfügung stehen.

In seinem Beitrag diskutiert Peter Preiß die Möglichkeiten der Förderung kaufmännischer Kompetenzen durch den wirtschaftsinstrumentellen Ansatz. Dabei überträgt Preiß das in der PISA-Studie verwendete mehrdimensionale Kompetenzmodell auf die kaufmännische Kompetenzentwicklung und zeigt anhand des Beispiels des Mo- dellunternehmens A \& S GmbH, einer Kettenfabrik, wie sich eine integrative Kompetenzförderung darstellen kann. Zentrales Element des Unterrichts ist die ökonomische Interpretation betrieblicher Leistungsprozesse anhand des angesprochenen Unternehmensmodells, mit dessen Hilfe die Marktbeziehungen des Unternehmens (Real- und Nominalgüterströme), der interne Leistungsprozess und die Bestände abgebildet werden. Die fachtheoretische Grundlage bildet der systemorientierte Ansatz der Betriebswirtschaftslehre. Dort wird die Unternehmung unter Rückgriff auf allgemeine systemtheoretische und kybernetische Vorstellungen als produktives soziales System gekennzeichnet und als strukturierter Sozialverbund zur Erzeugung von Marktleistungen betrachtet. Als wesentliche Punkte des Göttinger Ansatzes sind zu nennen:

- Die Modellierungsmethode zielt darauf ab, dass die Buchführung als zielbezogener Modellierungsprozess verstanden und vollzogen werden kann.

- Es findet ein permanenter Wechsel der medialen Repräsentationsformen statt: Neben konkret-gegenständlichen Objekten und Abläufen und der Arbeit mit umfassenden, didaktisch aufbereiteten Belegsătzen kommen verbale Vorgangsbeschreibungen zum Einsatz.

- Im Unterricht wird durchgängig mit einem Modellunternehmen gearbeitet; Geschäftsvorfälle sind unter Bezugnahme auf das allgemeine Unternehmensmodell zu interpretieren und zu buchen.

- Im Unterricht bearbeitet man mehrere, aufeinander aufbauende Geschäftsgänge mit wirtschaftlich sinnvollen Abläufen und Strukturen.

- Das externe Rechnungswesen wird als Controllinginstrument genutzt. Daten werden von Anfang an ausgewertet und interpretiert, Buchführungs- und Bilanzdaten durch Statistiken, Kennzahlen und verbale Berichte ergänzt. Zur Unterstützung der Einübung ökonomischer interpretationen sind Quartalsübersichten zu erstellen.

Tade Tramm zeigt in seinem Beitrag "Wirtschaftsinstrumentelles Rechnungswesen konkret" am Beispiel des bereits angesprochenen Lehrbuchs von Joost, Kripke \& Tramm (2002), wie sich die Konzeption des wirtschaftsinstrumentellen Rechnungswesens konkret unterrichtlich umsetzen lässt - quasi im Sinne einer praktischen Bewährungsprobe. Er stellt heraus, dass trotz einer grundsätzlichen Übereinstimmung der Göttinger und der Hamburger Variante des wirtschaftsinstrumentellen Rechnungswesens beide Vorgehensweisen $\mathrm{zu}$ teilweise unterschiedlichen fachdidaktischen Akzentsetzungen gelangen. Im Unterschied zu Preiß (1999) bzw. Getsch \& Preiß (2001) verzichtet Tramm darauf, die Lernsituationen aus dem kontinuierlich 
entwickelten Geschäftsablauf heraus zu gewinnen. Stattdessen werden jeweils spezifische Situationen skizziert, die in ein Gesamtszenario eingebettet werden. Hier wurde sicherlich ein Mehr an Flexibilităt für den Lehrenden gegen einen Verlust an Komplexität und Realitătsnăhe "eingetauscht". Folgerichtig wird daher im Lehrbuch im Anfangsunterricht kein auf Dauer angelegtes Unternehmen dargestellt. Vielmehr greift man auf ein „Auszubildendenprojekt" zurück, um betriebliche Wertschöpfungsprozesse anschaulich und gleichzeitig auf das Wesentliche konzentriert thematisieren zu können. Gemein sind beiden Varianten jedoch zentrale Konstruktionsprinzipien sowie insbesondere die Auffassung, dass dem Rechnungswesen für die Steuerung von Unternehmensprozessen eine zentrale Rolle zukommt.

Als Beitrag zur Überwindung der fehlenden empirischen Durchdringung des Rechnungswesenunterrichts sind die Ausführungen von Jürgen Seifried, Karsten D. Wolf, Christina Klüber und Detlef Sembill zu verstehen. Das Bamberger Forschungsteam hatte sich in Zusammenarbeit mit einer Bamberger Berufsschule seit Jahren intensiv mit der Erstellung und Überprüfung von Unterrichtskonzeptionen für den Rechnungswesenunterricht beschäftigt. Mittels einer aufwendigen empirischen Gegenüberstellung dreier Schulklassen einer Bamberger Berufsschule wurde überprüft, welche methodischen und curricularen Modellierungen sich günstig auf Lernprozess und Lernerfolg auswirken. Dabei zeigte sich, dass sich eine konsequente Schülerorientierung insbesondere dann als gewinnbringend emweist, wenn der Lernprozess durch adäquate und differenzierte Lernmaterialien unterstützt wird. Auf den Buchführungsunterricht gewendet bedeutet dieser pädagogische Allgemeinplatz, dass insbesondere die Kombination der Lehr-Lern-Konzeption des selbstorganisierten Lernens bei simultaner Orientierung an der Didaktik des wirtschaftsinstrumentellen Rechnungswesens zu einer signifikanten Verbesserung der Unterrichtsqualităt führt.

Weiterhin berichtet das Bamberger Forschungsteam über einen Weg, wie die in der Feldforschung gewonnenen Erkenntnisse in die Lehrerausbildung einfließen können. Hierzu stellen Karsten D. Wolf, Jürgen Seifried und Helge Städtler die Konzeption eines virtuellen Seminars zur Didaktik des Rechnungswesenunterrichts vor. Grundlegende Idee des Seminars ist, dass Studierende anhand einer konkreten Problemstellung die Planung und Vorbereitung sowie die Beurteilung von selbstorganisationsoffenem Unterricht selbst durchführen und theoriegeleitet reflektieren können. Der Online-Kurs wurde in der von Wolf und Stădtler entwickelten Lernumgebung EverLearn (http://www.everlearn.info) implementiert. Um den Lernerfolg der Studierenden sicherzustellen, wurde die Online-Arbeit durch Präsenzphasen ergänzt ("blended learning"), die Benutzerfreundlichkeit der Lernumgebung (Usability) evaluiert und bei Bedarf im Prozess verbessert. Als zentrale Gelingensbedingung von ELearning wird der Einsatz von geschulten Tutoren genannt, die Kommunikation, Kooperation und Kollaboration der Lernenden anleiten und unterstützen. Erste empirische Befunde belegen, dass das Seminar von den Studierenden gut angenommen wurde und dass sich die grundlegenden Konstruktionsprinzipien bewährt haben.

Lothar Reetz greift in seinem Beitrag das wichtige Thema der Überprüfung von Lernfortschritten und Lernergebnissen auf. Reetz beklagt die Einseitigkeit der bisher vorherrschenden Prüfungspraxis, bei der so genannte Situationsaufgaben lediglich eine nachrangige Rolle spielen. Mit Hilfe dieses Aufgabentypus gelingt es, so Reetz, reine Wissensreproduktion und punktuelle Veranschaulichung hinter sich zu lassen. Vielmehr geht es um die. Förderung von Handlungs- und Problemorientierung als Zielgröße des kaufmännischen Unterrichts. Diese erlebt gegenwärtig im Sog der Diskussion um die Handlungskompetenz bzw. deren Berücksichtigung als Zielkategorie in Prüfungsordnung eine Art Renaissance. Gleichzeitig warnt Reetz davor, das Konzept nicht in einer Weise zu modifizieren, die dem Postulat der Handlungskompetenz nicht mehr gerecht wird. Will man Lernaufgaben konstruieren, die Praxisbezug und authentischen Charakter aufweisen, so müssen Prüfungsaufgaben genau diesen Aspekten Rechnung tragen. Reetz optiert für die Verwendung offener Situationsaufgaben, bei denen der Prüfling aufgefordert ist, über das Verstehen der Situation problemlosend zu eigenen Ergebnissen zu gelangen.

Die Übersicht über die Beiträge des Sammelbandes zeigen, dass die Frage, wie ein qualitativ hochwertiger Rechnungswesenunterricht ausgestaltet sein soll, in Fachkreisen nicht unumstritten ist. Demgegenüber besteht weitgehend Konsens darüber, dass es zu einer Qualitätssteigerung des herkömmlichen Rechnungswesenunterrichts einer umfassenden Reform bedarf. Bisher erwies sich der Rechnungswesenunterricht jedoch als ăußerst veränderungsresistent (s. o.), und dies trotz vielfältiger - teilweise im vorliegenden Band dokumentierten - Impulse seitens der universitären Vertreter der Wirtschaftspădagogik. Im Zentrum der Diskussion steht dabei die Auseinandersetzung mit alternativen fachdidaktischen Konzeptionen. Fundierte empirische Untersuchungen zum Rechnungswesenunterricht liegen in weit geringerer Zahl vor, und diese befassen sich darüber hinaus mehrheitlich mit Lernschwierigkeiten (siehe hierzu Pawlik 1980, Tramm, Hinrichs \& Langenheim 1996 sowie Seifried 2004a). Empirische Gegenüberstellungen allgemeindidaktischer und/oder fachdidaktischer Ansätze fehlen jedoch bislang weitgehend (siehe auch Preiß 1999, S. 127) 
Darüber hinaus wissen wir nur wenig darüber, welche Einstellungen und Überzeugungen Fachlehrer zum Buchführungsunterricht haben. Neben der Ausarbeitung und Verfeinerung fachdidaktischer Konzeptionen sollte es den Wirtschaftspädagogen auch darum gehen, die empirische Basis des Wissens über den Rechnungswesenunterricht zu verbreitern und entsprechende Forschungslücke zu schließen.

\section{Literatur}

Achtenhagen, F. (Hrsg.) (1990): Didaktik des Rechnungswesens. Programm und Kritik eines wirtschaftsinstrumentellen Ansatzes, Wiesbaden: Gabler.

Achtenhagen, F. (1996): Entwicklung ökonomischer Kompetenz als Zielkategorie des Rechnungswesenunterrichts, in: Preiß, P. \& Tramm, T. (Hrsg.): Rechnungswesenunterricht und ökonomisches Denken. Didaktische Innovationen für die kaufmännische Ausbildung Wiesbaden: Gabler, S. 22-44.

Dubs, R. (1996): Historische und systematische Entwicklungslinien einer Didaktik des Rechnungswesens in der Schweiz, in: Preiß, P. \& Tramm, T. (Hrsg.): Rechnungswesenunterricht und ökonomisches Denken. Didaktische Innovationen für die kaufmännische Ausbildung, Wiesbaden: Gabler, S. 118-138.

Getsch, U. \& Preiß, P. (2001): Modellunternehmen A \& S GmbH - Grundkurs Rechnungswesen - belegorientiert Lern- und Lehrmaterialien für den wirtschaftsinstrumentellen Ansatz nach der Modellierungsmethode, Bad Homburg: Gehlen. CD-ROM.

Joost D., Kripke, G. \& Tramm, T. (2002): Wirtschaftsinstrumentelles Rechnungswesen, Troisdorf: Bildungsverlag EINS.

Pawlik, W. (1980): Fachdidaktik des Unterrichts in Rechnungswesen, in: Zeitschrift für Berufs- und Wirtschaftspädagogik, 76. Jg., H. 1, S. 33-43.

Philipson, J. J. M. (1813): Briefe über das kaufmännische Rechnungswesen, worin das einfache, das italienische, das englische und die vorzüglichsten neuern Systeme des Buchhaltens nach einer neuen und erleichterten Methode vorgetragen und einige neue Vorschläge, das italienische Buchhalten zu vereinfachen, ohne ihm etwas von seiner Zuverlässigkeit zu entziehen, mitgeteilt werden; besonders die, welche ohne Lehrer sich selbst unterrichten wollen, Hannover: Gebrüder Hahn.

Preiß, P. (1999): Didaktik des wirtschaftsinstrumentellen Rechnungswesens, München \& Wien: Oldenbourg

Preiß, P. (2000): Der Rechnungswesenunterricht als Beitrag zum Verständnis okonomische Zusammenhänge und wirtschaftlicher Entscheidungen, in: Bundesverband der Lehrer an Wirtschaftsschulen e.V. (Hrsg.): Funktionswandel des Rechnungswesens. Von der Dokumentation zur Steuerung, Sonderschriftenreihe des VLW, H. 44, Wolfenbüttel: Heckner S. $7-29$
Preiß, P. \& Tramm, T. (1990): Wirtschaftsinstrumentelle Buchführung. Grundzüge eines Konzeptes der beruflichen Grundqualifikation im Umgang mit Informationen über Mengen und Werte, in: Achtenhagen, F. (Hrsg.): Didaktik des Rechnungswesens. Programm und Kritik eines wirtschaftsinstrumentellen Ansatzes, Wiesbaden: Gabler, S. 13-94.

Preiß, P. \& Tramm, T. (1996): Die Göttinger Unterrichtskonzeption des wirtschaftsinstrumentellen Rechnungswesens, in: Preiß, P. \& Tramm, T. (Hrsg.): Rechnungswesenunterricht und ökonomisches Denken. Didaktische Innovationen für die kaufmännische Ausbilricht und ökonomisches Denken. Didakti
dung, Wiesbaden: Gabler, S. 222-323.

Reetz, L. \& Tramm, T. (2000): Lebenslanges Lernen aus der Sicht einer berufspädagogisch und wirtschaftspådagogisch akzentuierten Curriculumforschung, in: Achtenhagen, F. \& Lempert, W. (Hrsg.): Lebenslanges Lernen im Beruf - seine Grundlegung im Kindes- und Jugendalter, Band 5: Erziehungstheorie und Bildungsforschung. Opladen: Leske + Budrich, S. $69-120$

Reinisch, H. (1991): Ökonomisches Kalkül und kaufmännisches Selbstbild. Die Formung des kaufmännischen Arbeitsvermögens im vorindustriellen Deutschland unter besonderer $\mathrm{Be}$ rücksichtigung des Buchhaltungsunterrichts - zugleich eine Studie zu dessen Geschichte rücksichtigung des Buchhaltungsunterrichts - zugleich eine Studie zu dessen Geschichte-
und Didaktik von den Anfängen bis zum Ende des 18. Jahrhunderts als Beitrag zur berufspädagogisch-historischen Curriculumforschung, Habilitationsschrift, Universität Oldenburg.

Reinisch, H. (1996): "Leitbilder". Argumentationsmuster und curriculare Konstruktionen in der Didaktik des Rechnungswesenunterrichts. Eine historisch-systematische Analyse, in: Preiß, P. \& Tramm, T. (Hrsg.): Rechnungswesenunterricht und okonomisches Denken. Didaktische Innovationen für die kaufmännische Ausbildung, Wiesbaden: Gabler, S. 4584.

Reinisch, H. \& Struve, K. (2002): Was können wir aus der Geschichte beruflicher Arbeit und berufbezogener Didaktik lernen? Zur Bedeutung einer historischen Analyse/Synthese der
gegenständlichen Dimension ökonomischer und gewerblich-technischer Bildung, in: Eckert, M., Horlebein, M., Lisop, I., Reinisch, H. \& Tramm, T. (Hrsg.): Bilanzierungen. Schulentwicklung, Lehrerbildung und Wissenschaftsgeschichte im Feld der Wirtschaftsund Berufspädagogik, Frankfurt/Main: GAFB, S. 99-150.

Schneider, W. (2000): Didaktik des Rechnungswesens im Konflikt zwischen Fachwissenschaft, komplexer Realităt und subjektiver Faßlichkeit, in: Euler, D., Jongebloed, H.-C. \& Sloane, P. F. E. (Hrsg.): Sozialökonomische Theorie - sozialökonomisches Handein. Konturen und Perspektiven der Wirtschafts- und Sozialpädagogik, Kiel: bajOsch-Hein Verlag für Berufs- und Wirtschaftspädagogik, S. 191-210.

Schneider, W. (2002): Betriebliches Rechnungswesen für Einsteiger, 4. überarb. und erg. Aufl., Wien: Manz Verlag Schulbuch.

Seifried, J. (2004a): Fachdidaktische Variationen in einer selbstorganisationsoffenen Lern umgebung - Eine empirische Untersuchung des Rechnungswesenunterrichts, Wiesbaden: Deutscher Universitäts-Verlag.

Seifried, J. (2004b): Schüleraktivitäten beim selbstorganisierten Lernen und deren Auswirkungen auf den Lernerfolg, in: Zeitschrift für Erziehungswissenschaft, H. 4., 7. Jg., S. 571586 . 
Seifried, J., Brouër, B. \& Sembill, D. (2002): Was lernen Schülerinnen und Schüler im selbstorganisationsoffenen Rechnungswesenunterricht?, in: Zeitschrift für Berufs- und Wirtschaftspädagogik, 98. Jg., H. 4, S. 574-593.

Seifried, J. \& Sembill, D. (2004): Rechnungswesenunterricht neu gestalten - Empirische Befunde aus einem Kooperationsprojekt zwischen Schule und Hochschule, in: Wirtschaft und Erziehung, H. 11, 56. Jg., S. 371-379.

Sembill, D. (1992): Problemlösefăhigkeit, Handlungskompetenz und Emotionale Befindlichkeit. Zielgrößen Forschenden Lernens, Göttingen, Toronto \& Zürich: Hogrefe.

Sembill, D. (2000): Selbstorganisiertes und Lebenslanges Lernen, in: Achtenhagen, F. \& Lempert, W. (Hrsg.): Lebenslanges Lernen - seine Grundlegung im Kindes- und Jugendalter, Band 4: Formen und Inhalte von Lernprozessen, Opladen: Leske + Budrich, S. 60 90.

Sembill, D. (2004): Abschlussbericht zu "Prozessanalysen Selbstorganisierten Lernens" im Rahmen des DFG-Schwerpunktprogramms "Lehr-Lern-Prozesse in der kaufmännischen Erstausbildung". Bamberg. Download unter http://wipaed.sowi.uni-bamberg.de/awgs/ wipaed2002/download-pdf/abschlussbericht_prozess-analysen.pdf

Sloane, P. F. E. (1996): Didaktik des Rechnungswesens, Pfaffenweiler: Centaurus-Verlagsgesellschaft.

Terhart, E. (1997): Lehr-Lern-Methoden. Eine Einführung in Probleme der methodischen Organisation von Lehren und Lernen, 2., überarb. Aufl., Weinheim \& München: Juventa.

Tramm, T. (1992): Konzeption und theoretische Grundlagen einer evaluativ-konstruktiven Curriculumstrategie - Entwurf eines Forschungsprogramms unter der Perspektive des Lernhandelns. Berichte des Seminars für Wirtschaftspădagogik der Georg-August-Universität Göttingen, Band 17, Dissertation, Göttingen.

Tramm, T., Hinrichs, K. \& Langenheim, H. (1996): Lernschwierigkeiten im Buchführungsunterricht, in: Preiß, P. \& Tramm, T. (Hrsg.): Rechnungswesenunterricht und ökonomisches Denken. Didaktische Innovationen für die kaufmännische Ausbildung, Wiesbaden: Gabler, S. 158-221.

Tramm, T. \& Preiß, P. (1996): Rechnungswesenunterricht und ökonomisches Denken, in: Preiß, P. \& Tramm, T. (Hrsg.): Rechnungswesenunterricht und okonomisches Denken. Didaktische Innovationen für die kaufmännische Ausbildung, Wiesbaden: Gabler, S. 1-21. 